\title{
Filigrane
}

Écoutes psychanalytiques

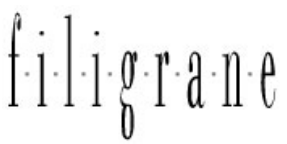

\section{Quatre femmes et leur contre-transfert}

\section{Daniella Angueli}

Volume 25, numéro 2, 2016

URI : https://id.erudit.org/iderudit/1039651ar

DOI : https://doi.org/10.7202/1039651ar

Aller au sommaire du numéro

\section{Éditeur(s)}

Revue Santé mentale au Québec

ISSN

1192-1412 (imprimé)

1911-4656 (numérique)

Découvrir la revue

\section{Citer cet article}

Angueli, D. (2016). Quatre femmes et leur contre-transfert. Filigrane, 25(2), 123-144. https://doi.org/10.7202/1039651ar

\section{Résumé de l'article}

Cet article vise à présenter les perspectives cliniques de quatre psychanalystes : Paula Heimann, Margaret Little, Annie Reich et Lucia Tower. À la même époque, elles se sont chacune prononcées sur la position de l'analyste à travers le concept de contre-transfert. Leur approche de travail, leur point de vue et leurs techniques sont aujourd'hui non seulement largement appliqués dans les psychanalyses de type psychothérapeutique, mais aussi adoptés comme ligne axiale dans les approches psychanalytiques de patients n'appartenant pas à la catégorie de la névrose « classique ». Bien qu'elles proviennent d'horizons théoriques différents, elles se rejoignent audacieusement sur une même idée : le contre-transfert est l'une des conditions nécessaires à la relation analytique et peut même devenir un outil précieux pour explorer l'inconscient de l'analysé. Ceci, naturellement, ne va pas sans risques. S'agirait-il d'approches caractérisant une position inconsciente féminine ? En mobilisant leurs propres possibilités émotionnelles d' "écoute ", en s'appuyant sur leur propre

inconscient et en conversant avec l'autre non névrotique de manière novatrice, elles ont essayé d'expérimenter d'autres formes de connexion avec l'autre de souffrance qu'est leur analysant.
Ce document est protégé par la loi sur le droit d'auteur. L’utilisation des services d’Érudit (y compris la reproduction) est assujettie à sa politique d'utilisation que vous pouvez consulter en ligne.

https://apropos.erudit.org/fr/usagers/politique-dutilisation/ 


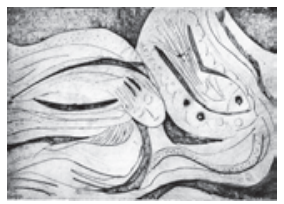

\section{Quatre femmes et leur contre-transfert}

\section{Daniella Angueli}

RÉSUMÉ: Cet article vise à présenter les perspectives cliniques de quatre psychanalystes: Paula Heimann, Margaret Little, Annie Reich et Lucia Tower. À la même époque, elles se sont chacune prononcées sur la position de l'analyste à travers le concept de contre-transfert. Leur approche de travail, leur point de vue et leurs techniques sont aujourd'hui non seulement largement appliqués dans les psychanalyses de type psychothérapeutique, mais aussi adoptés comme ligne axiale dans les approches psychanalytiques de patients n'appartenant pas à la catégorie de la névrose "classique». Bien qu'elles proviennent d'horizons théoriques différents, elles se rejoignent audacieusement sur une même idée: le contre-transfert est l'une des conditions nécessaires à la relation analytique et peut même devenir un outil précieux pour explorer l'inconscient de l'analysé. Ceci, naturellement, ne va pas sans risques. S'agirait-il d'approches caractérisant une position inconsciente féminine? En mobilisant leurs propres possibilités émotionnelles d' «écoute», en s'appuyant sur leur propre inconscient et en conversant avec l'autre non névrotique de manière novatrice, elles ont essayé d'expérimenter d'autres formes de connexion avec l'autre de souffrance qu'est leur analysant.

Mots clés: Paula Heimann, Margaret Little, Annie Reich, Lucia Tower, clinique, contre-transfert

$\mathrm{N}$ ous introduirons ici les points de vue de quatre psychanalystes, Paula Heimann, Margaret Little, Annie Reich et Lucia Tower, qui, vers la même époque, dans les années 50, ont exposé leurs idées quant à la position de l'analyste à travers le concept de contre-transfert. Leur approche de travail, leur point de vue et leur technique sont aujourd'hui non seulement largement appliqués dans les psychanalyses de type psychothérapeutique, mais aussi adoptés comme ligne axiale dans les approches psychanalytiques (même autres que celles anglo-saxonnes) de patients n'appartenant pas à la catégorie de la névrose «classique»-si tant est que ce terme a encore cours. S’agit-il d'approches caractérisant une position inconsciente féminine qui, justement, les facilite?

À cette époque, et plus tard également, l'idée que ces quatre analystes se faisaient du contre-transfert et de la manière de l'utiliser pendant la cure analytique allumait en France des foyers de dissension entre psychanalyse 
lacanienne et psychanalyse anglaise/post-kleinienne. Ces idées ont donné à Lacan lui-même et plus généralement aux analystes lacaniens l'occasion dont ils avaient besoin pour expliquer leurs positions quant à la place de l'analyste dans la thérapie, par rapport au transfert et au-delà de l'autre spéculaire/ imaginaire. C'est dans ce contexte, au plus fort du conflit, que Lacan a conçu l'idée de l'«objet a d dans l'analyste et proposé le «désir de l'analyste» comme réponse alternative au transfert de l'analysant, au-delà du contre-transfert.

Cette nouvelle approche, dont ces quatre analystes sont à l'époque les chefs de file, prendra peu à peu de l'importance dans l'histoire de la psychanalyse et, avec le temps, influencera diverses orientations de la géographie psychanalytique. Un exemple en est, dans les années 90 à New York, l'école dite «intersubjective», qui utilisera les propositions du courant du contretransfert en leur attribuant des désignations comme «empathie», «insight intersubjectif» et «co-création», dans un effort de faire progresser les analyses et thérapies de pathologies «difficiles» - états limites ou cas de délinquance - demandant un traitement thérapeutique différent.

\section{« Pour-transfert » féminin}

Après tant de décennies d'existence de la psychanalyse, nous avons bien assimilé l'idée que les analyses sont multiples et qu'elles se transforment à chaque époque. De nos jours, la société moderne les confronte à d'autres répétitions et à des jouissances étranges, inexistantes il y a 50 ans.

À une époque où l'on discourt sur les affects dans de doctes conférences et où ceux-ci font l'objet d'études dans les départements psychosociaux des universités (comme s'il était besoin de prouver qu'il existe encore des sentiments), à une époque où la psychanalyse a été, à d'innombrables reprises, la cible d'exorcismes scientifiques de tout acabit et que les psychothérapies «abrégées», les méthodologies «technocratiques» et "scientificiste» promettent d'acquérir vite, facilement et à bon marché ce au sujet de quoi la psychanalyse classique jurait qu'il valait mieux assumer l'impossibilité de l'atteindre, à l'époque du grand Autre transmuté et du «stade de l'écran» qui ne se contente pas de refléter notre adversaire imaginaire, mais nous appelle à nous noyer en le regardant, il peut paraître utile de nous rappeler que ces quatre analystes ont eu l'audace d'expérimenter d'autres formes de connexion avec l'autre de souffrance qu'est leur analysant. En mobilisant une série de leurs propres possibilités émotionnelles d' "écoute», en se servant de leur propre inconscient, en conversant avec l'autre de manière différente quand ce dernier n'est pas capable de répondre à un désir d'analyse approprié, 
parce que non névrotique, elles ont essayé, expérimenté, elles ont porté le "petit autre», souhaitant déclencher une autre dynamique qui combattrait l'immobilité du symptôme ou sa persistance intransigeante. Art ou artifice? Voilà ce que répondent, en appliquant leur propre méthode, ces dames de la psychanalyse. Leur réponse serait-elle caractéristique du sexe féminin?

\section{Paula Heimann : deux inconscients en consonance}

Paula Heimann, psychanalyste allemande ayant initialement appartenu au groupe des analystes kleiniens, a travaillé aux côtés de Melanie Klein et noué avec elle une relation très personnelle. À la suite d'un violent désaccord, Heimann s'est séparée de Melanie Klein pour suivre, auprès de Donald Winnicott, un parcours théorique indépendant des principes de la Société britannique de psychanalyse.

Dans son article très controversé de 1949, «À propos du contre-transfert», elle présente sa conception du positionnement de l'analyste dans le cadre psychanalytique et définit le type de relation que ce positionnement établit avec l'analysant. Pour certains, c'est l'exemple à ne pas suivre. Pour d'autres, c'est une proposition. Pour son époque, en tout cas, c'était l'expression d'une psychanalyste résolument «indépendante».

Heimann, en règle générale, voyait dans le cadre psychanalytique deux inconscients, l'un vibrant dans l'autre, sans pour autant que les conditions de l'un s'identifient à celles de l'autre. C'est dans cette optique qu'elle élabore sa conception de ce qu'est le contre-transfert, terme qu'elle emploiera pour désigner «la totalité des sentiments que l'analyste éprouve envers son patient» (Heimann, 1949). Sa thèse est que le contre-transfert est, à l'intérieur de la situation analytique, un instrument précieux, grâce auquel l'analyste est à même d'interpréter l'inconscient du patient. Le psychanalyste n'est pas un cerveau mécanique qui effectuerait un travail purement intellectuel pour émettre ses interprétations. La situation psychanalytique est décrite par Heimann comme une relation entre deux personnes qui éprouvent des sentiments; la seule distinction tenant au fait que l'analyste doit pouvoir soutenir les sentiments éveillés en lui, afin de les subordonner au travail analytique. Dans ce travail, il doit fonctionner comme le "miroir réfléchissant» du patient. À côté de l'attention flottante régulière, l'analyste dispose d'une sensibilité émotionnelle vibrant librement, qui l'aide à suivre les mouvements émotionnels du patient. Selon Paula Heimann (1949), «l'inconscient de l'analyste comprend celui de son patient». Cette «relation profonde» s'exprime dans les sentiments composant le contre-transfert de l'analyste. Le 
psychanalyste trouve alors un indice de la justesse de sa compréhension, en comparant ses propres sentiments aux associations et aux comportements du patient. S'ils concordent, c'est que l'analyste a atteint une compréhension véritable.

Heimann ajoute qu'il est bien sûr toujours possible que surgissent des sentiments très intenses, qui altèrent l'observation et la juste évaluation des faits, car ils incitent davantage à l'action qu'à la réflexion. C'est la raison pour laquelle la sensibilité émotionnelle de l'analyste devra être "plutôt extensive qu'intensive, différentiative et mobile». En général, si l'analyste combine l'attention libre avec les libres réponses émotionnelles, ses sentiments ne feront pas obstacle, parce qu'ils s'accorderont avec ceux qu'il comprend dans l'inconscient du patient.

Toutefois, ce à quoi Heimann attachera la plus grande importance est davantage la «perception inconsciente» de l'inconscient du patient que la conception consciente de la situation.

Il en ressort que la mobilité inconsciente de l'analyste, lors de son contact avec le patient, est d'une importance capitale. Le contre-transfert en vient même à être caractérisé de "création du patient», faisant partie de sa personnalité. Nous comprenons immédiatement que l'analyste établit un équilibre sur les lieux d'une dualité, à laquelle il présente son récepteur inconscient en correspondance naturelle avec un mouvement inconscient se produisant dans l'autre. Pour quelle raison? De quoi souffre l'analysant? Quelle est sa relation avec la parole et quels sont les obstacles, pulsionnels ou autres, qui s'opposent à l'expression et conduisent l'analyste à recourir aux perceptions inconscientes afin de comprendre les émissions de l'autre? Telles sont, pensons-nous, les questions les plus pertinentes soulevées par une telle position dans laquelle, semble-t-il, le psychanalyste prend le risque d'imputer au patient ce qui lui appartient en propre. Heimann croit néanmoins qu'un tel risque est inexistant du moment que l'analyste, au cours de sa propre analyse, a travaillé sur ses conflits infantiles et ses angoisses (paranoïdes et dépressives) jusqu'à pouvoir aisément établir le contact avec son propre inconscient. Cela lui évitera de devenir acteur dans chaque scène que le patient rejoue dans la relation analytique.

Par conséquent, il existe une condition qui, d'après cette théoricienne, est d'une importance primordiale dans ce contact d'inconscient à inconscient: une très bonne analyse du monde archaïque de l'analyste. C'est ce qui lui permet de sortir de la position de semblable et lui donne sur le terrain la place de récepteur averti. 
Le point de vue de Heimann est intéressant, du fait que la réponse émotionnelle de l'analyste est non seulement une clé permettant d'ouvrir l'inconscient du patient, mais peut aussi être une «stimulation à assumer son propre travail et à poursuivre l'analyse de ses problèmes». Bien entendu, on peut se demander en ce point quelle priorité elle donnait, pendant la pratique analytique, aux élaborations de l'analyste le concernant en propre.

Quoi qu'il en soit, d'après Heimann, l'analyste ne doit pas et n'a pas le droit de communiquer ses mouvements intérieurs au patient, comme le proposait Ferenczi dans son «analyse mutuelle». Chez Heimann, la communication entre les deux inconscients a toujours certaines limites et conditions quant à la participation de l'analyste à l'intérieur de cet environnement inconscient. L'analyste est conscient de la difficulté qu'il y a à délimiter le terrain où il évolue. Cependant, elle insiste sur ce point. De toute évidence, elle croit qu'il est besoin d'outils qui ouvrent la voie de l'expression à des éléments dans l'autre qui ne sont pas associés à des signifiances verbales, ni (encore?) saisissables dans des signifiances; donc à des éléments immobilisés d'actes pulsionnels et peut-être d'instants traumatiques. C'est là sa façon de remobiliser les inerties répétitives incorporées dans le transfert, dans le non dialectique et dans le symptôme, qui n'est pas explicitement (ou seulement) névrotique. Et Heimann donne l'impression de n’hésiter en rien quant à cette question, cette posture ou ce postulat étant sa dynamique particulière sur le terrain. Pourrait-on dire qu'elle établit un autre type de relation à l'inconscient déjà là de l'autre, déjà inscrit, déjà décidé à ne pas se permettre de se manifester?

\section{Margaret Little : la résistance du contre-transfert}

Membre titulaire de la Société britannique de psychanalyse depuis 1945, cette psychanalyste anglaise a également été membre du Middle Group dont les positions théoriques différaient de celles du groupe de Melanie Klein et de celui d'Anna Freud.

Margaret Little est présentée comme étant favorable à une intense participation de l'analyste à chaque expression inconsciente de l'analysant, même dans le champ du vécu. Patiente, entre autres, de D. Winnicott, et ayant, selon elle, traversé des expériences de l'ordre du borderline, elle élaborera une approche audacieuse des patients atteints de cette pathologie, mais aussi des psychotiques, et formulera des points de vue qui susciteront des réactions nombreuses et variées. Aussi étrange que puisse nous paraître une théorie permettant une inter-réaction démesurée entre analyste et analysant, il est 
cependant intéressant de voir formulés certains positionnements inconscients possibles de l'analyste, dont aucun silence psychanalytique ne saurait effacer l'effet sur le patient.

En 1951, dans son article «Le contre-transfert et la réponse qu’y apporte le patient», elle affirmera qu'à son avis la plupart des psychanalystes se tiennent dans une position phobique, voire paranoïde, envers leurs propres sentiments et idées pendant la cure, envers ce qui compose leur contretransfert. Alors qu'ils connaissent et reconnaissent son existence, ils évitent de l'interpréter, car ils le considèrent comme dangereux. Little est d'opinion différente. Tout d'abord, elle voit les deux participants à la cure comme inséparables. Elle en conclut que transfert et contre-transfert sont tout aussi inséparables. Et, si l'analyse et le bon usage du transfert constituent un instrument thérapeutique précieux, le bon usage du contre-transfert peut avoir les mêmes bons résultats dans le progrès de la psychanalyse, surtout lorsque celle-ci se trouve en cause.

Little nous invite avant tout à donner une définition claire du contretransfert, avant de le bannir comme dangereux: si le contre-transfert est un produit de la part inconsciente du moi de l'analyste, soit celle qui est la plus proche du ça, il existe néanmoins d'autres activités du moi, non refoulées, qui jouent un rôle dans le développement du contre-transfert, telles que la synthèse et l'intégration. Ainsi, Little présente le contre-transfert comme le résultat de la synthèse de divers lieux psychiques de l'analyste, alors qu'il avance aux côtés de l'analysant. Mais ce n'est pas tout. Pour elle, les limites entre les diverses dimensions psychiques sont très floues. En effet, elle croit que la tendance à distinguer le conscient de l'inconscient et l'inconscient refoulé de l'inconscient non-refoulé - alors que, en réalité, les éléments de chacun de ces lieux psychiques coexistent - fait obstacle à la définition du contre-transfert et à son bon usage.

En ce point, nous devons bien sûr nous demander dans quelle mesure chaque terme technique psychanalytique a le même sens pour toute la communauté analytique; il est nécessaire de tenir compte du sens qu'il revêt pour chacun, de la tradition analytique d'où il provient et, surtout, des patients qu'un théoricien a en tête lorsqu'il fait une proposition ou une construction théorique.

Dans la théorie de Little, analyste et analysant se trouvent psychiquement très proches, à portée de souffle. En effet, à son avis, la force motrice d'une analyse, ce qui incite irrésistiblement le patient à aller mieux, sont les exigences combinées du ça du patient et de celui de l'analyste, à la 
différence près que celles de l'analyste sont plus «dirigées» et plus «efficaces ", puisqu' elles sont le résultat de sa propre analyse.

Selon la conception de la relation psychanalytique formulée par Little, l'analyste doit nécessairement s'identifier au patient. Il peut s'identifier consciemment et inconsciemment au désir du moi du patient d'aller mieux, mais, de la même manière, il peut également s'identifier avec le surmoi et le ça du patient, de sorte qu'il peut aboutir au résultat contraire. Il s'agit de cas où, par exemple, l'analyste refuse inconsciemment l'amélioration de l'état du patient. Little parlera de contre-transfert débouchant sur une «contrerésistance». Pour que l'identification de l'analyste avec l'analysant n'aboutisse pas à ces résultats déplaisants, elle devra être suivie d'un détachement: «Le détachement est produit, au moins partiellement, en utilisant cette fonction du moi de tester la réalité, et en y introduisant les facteurs de temps et de distance» (Little, 1951). Cela signifie que, en ce qui concerne le temps, le patient ressent le passé comme un présent, tandis que l'analyste sait qu'il s'agit du passé; le facteur de la distance concerne le fait que l'expérience en question est celle du patient et non celle de l'analyste. Si l'analyste peut stabiliser - continue Little - cet intervalle de temps et de distance, l'analysant ne se heurte à aucun obstacle l'empêchant d'accomplir le cycle de son expérience dans son immédiateté, pour la laisser par la suite devenir passé, puisqu'il s'identifie à l'analyste qui, toutefois, lui permettra d'être psychiquement séparé de lui-même. Le progrès de la cure dépend en ce sens du rythme alterné d'identification et de séparation. Cependant, s'il ne tient pas compte de ces deux dimensions du temps et de la distance, l'analyste devient «l'aveugle qui conduit l'aveugle».

La clinique psychanalytique relève chez Little d'un art véritable où un régulateur sensible de la distance, du temps et du rythme dirige l'œuvre, tout en progressant lui-même dans cette œuvre. Pas facile. Risqué. Mais, ayant vécu, accompagnée par Donald Winnicott (Little, 1985), des expériences très proches de la psychose, ayant vu son analyste évoluer aux limites du symbiotique qu'elle réclamait de lui sans jamais qu'il n'entre en fusion avec elle, Little croit que, dans des situations cliniques si difficiles, il faut que l'analysant achève d'abord les cycles de ses besoins avec une mère good enough et que les processus psychotiques s'apaisent en lui, avant toute autre approche.

En effet, dans les cas de psychose, l'expérience de l'identification mutuelle joue, selon Margaret Little, un rôle particulièrement important. Du côté de l'analyste, il existe une tendance à s'identifier au ça du patient, peut-être en l'absence d'un moi intégré. D’ailleurs, plus la personnalité du patient sera 
désintégrée, plus elle deviendra un stimulus puissant des points les plus profondément refoulés de l'analyste, en appelant ainsi ses mécanismes de défense les plus primitifs à entrer en jeu.

Pour certains théoriciens-analystes, cette méthode pourrait impliquer un risque énorme. Little, pour sa part, semble plutôt la voir comme un défi. Quoi qu'il en soit, l'adoption de cette position révèle l'immense confiance que Little place dans l'analyse des mécanismes de défense primitifs de l'analyste, confiance qui ne tourne jamais vers le côté de la toute-puissance, mais qui lui permet de soigner son patient de manière différente.

De l'autre côté, il se peut qu'un fragment du moi brisé du patient s'identifie avec le moi du thérapeute et, de cette façon, établisse un premier contact avec la réalité à travers le contact de l'analyste avec elle. Chez le patient, se déclencherait alors un processus d'introjection progressive du monde extérieur, suivi d'une re-projection, cycle dans lequel l'énergie d'investissement provient de la libido du thérapeute.

Bien sûr, ce contact avec la réalité suppose un contact constant avec le thérapeute. Le patient s'autonomise très difficilement et toujours proportionnellement au degré de sa désintégration. Little croit que, dans le cas de patients hautement désintégrés, s'insinue le besoin de se servir du contretransfert, qui aide l'analyste à trouver chez le patient les éléments qui favoriseront le contact entre lui et son patient. Comment parvenir à ce résultat? «[... Le thérapeute doit permettre à ses idées et aux satisfactions libidinales issues de son travail de régresser jusqu'à un niveau extraordinairement bas » (Little, 1951).

Par conséquent, la seule façon pour le patient de sortir de l'enfer de sa désorganisation psychotique est que l'analyste y descende avec lui et qu'ils en reviennent ensemble.

Cette tactique a donné des résultats thérapeutiques exceptionnels qui, d'après la psychanalyste, ont été obtenus par: a. des analystes débutants qui, faute d'expérience, ne craignent pas de se laisser aller librement à leurs mouvements inconscients, et chez lesquels les éléments qui prédominent durant la cure sont les sentiments positifs; b. des analystes expérimentés qui se fient à leurs mouvements inconscients et peuvent amener leur contre-transfert à la conscience à tout moment. Ces analystes, capables de descendre aux enfers et d'en revenir intacts, seront, aux moments difficiles, aidés par le patient lui-même qui, comme l'analyste, constitue un miroir de l'autre.

Dans cet esprit de réciprocité, grâce auquel la relation psychanalytique devient perceptible, Little exhortera les psychanalystes, en cas d'erreur 
d'interprétation ou autre erreur technique, à reconnaître leur erreur et même à parler de leur contre-transfert au patient, parce que, de cette façon, ils lui montrent la subjectivité des sentiments, sans pour autant qu'il soit nécessaire de révéler leur provenance plus profonde. Cette mention du contre-transfert, soutient-elle, devra être «légère» et constituer un moyen d'atténuer la tension et l'angoisse du patient. Par ailleurs, elle affirme que l'analysant est doté d'une sensibilité naturelle au manque de sincérité de son analyste. Ainsi, si l'analyste ne reconnaît pas sa peur d'être asservi aux sentiments qu'il éprouve à l'égard du patient, ce dernier tendra également à refuser ses sentiments en s'identifiant avec l'analyste, ce qui nuira à l'analyse.

L'interprétation du contre-transfert convient surtout aux dernières phases de la psychanalyse, alors que «la capacité du patient à l'objectivité est suffisante » et a atteint le point où le patient découvre lui-même à l'analyste son contre-transfert. D'après Little, c'est une manière d'amener le patient, non seulement à reconnaître les comportements parentaux irrationnels qui ont joué un rôle important dans la formation de sa névrose, mais aussi à comprendre que ces comportements ne le visaient pas personnellement.

De plus, une fois que le moi du patient aura admis que l'analyste éprouve des sentiments inconscients, cela aidera à la levée du refoulement en portant à sa conscience des idées et des souvenirs jusque-là inaccessibles.

Little propose par ailleurs d'étendre les interprétations du transfert à l'interprétation de l'éventuel contre-transfert. D'après elle, cela libère les contre-transferts et les rend utilisables dans l'analyse. Or, la non-reconnaissance du contre-transfert peut aboutir à l'interruption prématurée de l'analyse ou à son prolongement, au re-refoulement de tout ce qui aurait pu devenir conscient.

Il ne fait donc aucun doute que cette théoricienne propose à l'analyste diverses modalités de rejet de la position de l'Autre absolu pendant la cure, étant donné qu'il évolue dans les géographies sensibles du psychotique. Cette implication de l'analyste vise clairement: $a$. à atténuer l'angoisse; $b$. à réduire au minimum chez l'analysant toute méfiance risquant de conduire à des crises de type psychotique; et $c$. à présenter peu à peu des élaborations signifiantes qui puissent nouer entre eux le réel, l'imaginaire et le symbolique. Dans ces élaborations, l'analyste s'offre en tant que compagnon de voyage et revient en toute occasion à ce positionnement. De là, nous comprenons les difficultés des patients auxquels il s'adresse. 
En 1956, Margaret Little introduit un autre terme ou, plutôt, un autre symbolisme à la place du terme de «contre-transfert» utilisé jusque-là. Il s'agit $\mathrm{du}$ « R». Le «R» donc, symbolise «la réponse totale de l'analyste se reportant aux besoins de son patient, quels que soient les besoins, et quelle que soit la réponse». À son avis, le contre-transfert, qui concerne les éléments refoulés non analysés chez l'analyste se reportant sur son patient, ne constitue qu'une partie du «R».

" "R", dès lors, comprend tout ce qui est conscient et tout ce qui est inconscient - tout ce qui est inconscient consistant en ce qui est refoulé (normalement ou pathologiquement), et bien des choses qui n'ont jamais été conscientes. En d'autres termes, "R" comprend des éléments qui appartiennent à la fois au moi, au surmoi et au ça de l'analyste» (Little, 1956). En disant «réponse totale», M. Little précise qu'elle entend «tout ce qu'un analyste dit, fait, pense, imagine ou ressent au cours de l'analyse, relativement à son patient». L'action de l'analyste ne se limite nullement à l'interprétation. Les réponses de l'analyste peuvent revêtir toutes sortes de formes: un comportement, la forme qu'il donne à son interprétation, les conditions qu'il définit pour le patient, un silence, une écoute, une réaction ou une non-réaction. Tout cela constitue l'expression des sentiments, conscients ou inconscients, de l'analyste.

Si cette réponse de l'analyste est inévitable, c'est qu'il existe un lieu où le patient et l'analyste nouent une relation d'égal à égal. Plus précisément, l'analyste se devra de répondre à la présence de l'analysant, non seulement dans le champ symbolique, mais aussi dans le champ de l'affectivité, de l'imaginaire et de chaque acte suscité par l'inconscient. Sa réponse est totale, parce que chaque mouvement du moi, du surmoi ou du ça chez l'analyste permet de participer d'une manière ou d'une autre à la situation psychanalytique.

La différence entre les deux protagonistes semble se fonder sur le point suivant: même si l'expression des sentiments, mais aussi l'expression de tout autre mouvement intérieur ou réaction de l'analyste émane de l'inconscient, on lui suppose toutefois une prédisposition consciente. Elle constitue un «acte de contact», dirions-nous, une stimulation qui donne à l'analysant la capacité - et le désir - d'approcher la réalité de l'autre et, par conséquent, la sienne propre, de se familiariser avec elle et de pouvoir la négocier par la parole, exactement comme le fait l'analysant et à travers l'identification avec l'analyste. D'ailleurs, dans la théorie de Little, la capacité de l'analysant à s'identifier avec l'analyste dans ses positions et dans sa responsabilité joue un rôle très important. 
En ce point, il serait bon de se demander encore une fois quel type de patients l'auteur avait en tête quand elle exprimait ces points de vue. Remarquons qu'elle fait souvent référence aux névroses de caractère, aux borderline et aux psychopathes. Dans le cadre psychanalytique, ces patients présentent des besoins nettement différents et leur ouverture dans le champ exclusif du symbolique est à examiner. D'après Margaret Little, les patients souffrant d'angoisses psychotiques ou de vraies psychoses ont besoin d'un contact "plus direct» avec l'analyste. Little considère que l'expression des sentiments et des réactions de l'analyste ouvre une voie vers l'interprétation, parce qu'elle rend ces patients accessibles, du fait qu'ils établissent un contact «dans une aire nouvelle».

À notre avis, il ne s'agit toutefois peut-être pas tant d'une «aire nouvelle» que d'une ouverture de l'analyste à l'intérieur du champ qui, chez l'analysant, lui avait toujours été connu, mais était resté muet jusque-là. La psychanalyste considère que la position de l'analyste comme «écran impersonnel» ou comme "miroir» est utile pour isoler le transfert chez les patients névrosés. Il reste cependant toujours la possibilité que les deux protagonistes l'utilisent finalement comme défense. En tout cas, il est important que l'expressivité, de la part de l'analyste, soit perçue comme une étape intermédiaire dans un trajet devant aboutir à l'interprétation. Autrement, l'éveil de ce champ entraînerait l'augmentation des résistances du patient. Cependant, pour que les interprétations de transfert puissent signifier quelque chose pour le patient, il faudra d'abord que son angoisse paranoïde s'allège et que soient rendus possibles le développement d'une relation, la capacité de supporter la fusion et la séparation qui s'y implique, ainsi que le risque de provoquer des sentiments chez l'autre ou d'éprouver lui-même des sentiments pour quelqu'un d'autre.

Toutefois, l'analyste peut lui-même nuire à l'évolution de l'analyse, s'il ne reconnaît pas ses sentiments envers le patient ni l'éventuelle culpabilité que ces sentiments suscitent en lui. En ce cas, ce sont ses résistances à lui qui sont en jeu. Little considère que la seule manière pour que l'analyste ait un bon «contact inconscient» avec le patient et qu'il n'entrave pas l'évolution du processus psychanalytique par son propre contre-transfert est l'autoanalyse permanente.

En général, le psychanalyste devra accepter d'utiliser, au cours de la cure, quelques surgissements de son propre inconscient. En particulier avec des patients proches de la psychose ou psychotiques, l'inconscient de l'analyste est présenté comme le premier instrument permettant d'approcher, de la 
manière la plus authentique, les processus primaires dans lesquels vit le patient. Bien plus tard, le moi du patient sera rendu plus accessible à la présence réelle de l'analyste, à la réalité même et, par la suite, aux interprétations du transfert.

Little croit que, lorsque l'analyste commence à faire confiance à ses propres processus inconscients, sa «contre-résistance» s'amoindrit plus rapidement. Et, étant donné que l'analyste est vu comme participant, une grande importance est accordée à sa capacité de négocier ses propres angoisses paranoïdes ainsi que sa dépression. Cette capacité le rendra - selon Little - assez sain et chaleureux pour briser le transfert illusoire des patients perturbés, en présentant la réalité «d'une manière comparable à l'éveil d'un rêve» (1956).

Pourrait-on dire qu'il s'agit d'une approche qui efface les différences et les délimitations entre les instances telles que conscient/inconscient, interprétation/comportement, analyste/analysant? Il s'agit certainement d'une technique époustouflante, dans la mesure où l'analyste, acharné à refuser les attributions du grand Autre de son analysant/patient, plonge avec lui dans une tentative d'auto-reconnaissance face à l'autre, en vue de l'aider à se reconnaître lui-même, à éprouver de la confiance pour le processus et à se laisser soulager par l'offre d'une réciprocité, dont le but est d'éviter l'angoisse. Il semble que Little ressente une confiance inébranlable dans cette technique. Elle a pour ainsi dire foi en ce qu'elle fait. Et il est bon d'écouter les analystes qui ont cette foi - extrêmement importante lorsqu'elle est offerte à un patient particulièrement désorganisé.

\section{Annie Reich : I'insight, l'acting-out et l'identification de I'analyste}

Vers la même époque, une autre théoricienne, l'Autrichienne Annie Reich, se penche elle aussi sur la question du contre-transfert. Analysée par Wilhem Reich (qu'elle épouse par la suite), Herman Nunberg et, plus tard, par Anna Freud, elle a été, entre autres, membre de la Société viennoise de psychanalyse jusqu'en 1938. Cette année-là, elle émigre aux États-Unis où elle rejoint la Société psychanalytique de New York, ainsi que l'Association internationale de psychanalyse dont elle reste un membre actif jusqu'à sa mort.

En 1951, elle publie son article «Sur le contre-transfert», où s'exprime peut-être la problématique générale qui prévalait, à ce moment-là, relativement à cette question. Nous nous trouvons devant un analyste qui, par 
définition, utilise son inconscient pour comprendre l'analysant, et non pas tant «ses processus actifs de pensée» et ses «conclusions logiques». A. Reich est partisan de l'«insight», de cette «claire appréhension du matériel» qui surgit subitement chez l'analyste et donne un sens à tout ce qui, jusqu'à cet instant, lui semblait incompréhensible et confus. Cet événement de clairvoyance qui survient au moyen de l'inconscient de l'analyste, ressemble à une identification brève et partielle au patient. Ensuite, l'analyste doit revenir à sa "position d'extériorité», de sorte à pouvoir évaluer objectivement ce qu'il vient de ressentir. En général, il devra pouvoir rester neutre, afin de faciliter le transfert et parvenir, d'une part, à se sentir profondément à l'intérieur d'une autre personne et, d'autre part, à ne pas s'impliquer. Ses sentiments et ses attitudes à l'égard du patient ne font pas obstacle, pourvu qu'ils soient conscients. Si, cependant, ils sont trop intenses, c'est qu'ils émanent de l'inconscient de l'analyste et, par conséquent, nous nous trouvons alors dans le champ du contre-transfert.

D'après A. Reich (1951), le contre-transfert est constitué «des effets, des besoins et conflits inconscients de l'analyste sur sa compréhension ou sa technique». Elle placera cependant ce contre-transfert sous le concept plus large de l'acting-out de l'analyste: "Nous parlons d'acting-out chaque fois que l'activité d'analyse a une signification inconsciente pour l'analyste. Alors, sa réponse au patient, et souvent tout le maniement de la situation analytique, seront motivés par des tendances inconscientes cachées». Dans les cas d'acting-out, le patient n'est pas l'objet sur lequel l'analyste transfère quelque chose de lui, mais l'instrument par lequel l'analyste satisfait certains de ses besoins, par exemple, apaiser son anxiété ou maîtriser ses sentiments de culpabilité.

A. Reich donne ce faisant quelques exemples de contre-transfert:

- L'analyste est envahi de sentiments (sympathie ou antipathie) à l'égard du patient, en raison de son identification avec lui. Le contretransfert devient en fait la manifestation de la défense de l'analyste contre une impulsion.

- Le contre-transfert dérive tout droit d'une impulsion.

- Le contenu spécifique du matériel du patient provoque chez l'analyste la réaction de défense que celui-ci avait développée, dans des situations similaires, durant son enfance.

- La relation analytique comme telle et quelques aspects particuliers de la relation au patient suscitent, chez l'analyste, des manifestations contre-transférentielles, par exemple une surévaluation des 
résistances du patient et un sentiment de ne pas être capable de les affronter et de les analyser.

- Le contre-transfert traduit un problème plus général de l'analyste, par exemple une agression inconsciente, et la culpabilité concomitante rendent l'analyste hésitant, trop conciliant et incapable de se tenir dans une position inflexible, quand il en est besoin. D'autres fois, l'analyste a la tendance paranoïde à chercher en l'autre les traces de traits qu'il possède lui-même à un degré élevé, en projetant, en fait, ses propres contenus inconscients sur les patients. Toutefois, il existe aussi une position contraire: l'analyste, pour maitriser son angoisse devant les surgissements émotionnels ou les explosions chez son patient, se tient, toujours selon A. Reich, dans une attitude d'isolement intellectuel devant l'inconscient et s'adonne à le "comprendre». Dans ce cas, le champ analytique en vient à constituer un terrain propice pour l'acting-out de l'analyste.

- Dans la même catégorie que l'acting-out de l'analyste figure le cas de l'analyste entretenant une mauvaise relation avec son propre inconscient. En pratique, cela se traduit par une tension de l'analyste dans son rapport au matériel inconscient du patient et par une difficulté, voire une peur, de formuler certaines interprétations, sans, auparavant les avoir pensées et analysées lui-même minutieusement et en profondeur.

- D'autres fois, l'analyste utilise l'analyse pour se procurer assurance et satisfaction narcissiques. Un exemple en est la position dite du «doigt de Midas», où l'analyste croit être un guérisseur magique qui peut restaurer la puissance et annuler la castration, ce qui lui donne une énorme satisfaction. Naturellement, une telle position lui ôte la capacité d'observer calmement, si bien qu'il aboutit facilement à une fausse évaluation de ses patients, laquelle engendre souvent une hostilité envers ceux qui ne parviennent à lui procurer la satisfaction narcissique en étant guéri par lui.

— Les attitudes "pédagogiques» des analystes qui, au lieu d'analyser l'angoisse de leurs patients, ont tendance à assouvir leurs désirs infantiles, restés insatisfaits, et à leur « enseigner » que le monde n'est pas aussi terrible que le présentait leur mode de pensée infantile.

Dans tous ces cas, l'analyse est utilisée à des fins inconscientes et avant tout «pour maintenir l'équilibre interne de l'analyste». A. Reich croyait que, d'habitude, les difficultés contre-transférentielles rencontrées chez les 
analystes sont de type «chronique» et résultent « des difficultés de personnalité profondément enracinées de l'analyste». À son avis, les difficultés aiguës sont beaucoup plus faciles à résoudre que les chroniques. Toutefois, dans les deux cas, il n'y a, selon elle, qu'un seul antidote: l'analyste doit poursuivre sa psychanalyse. N'oublions pas, d'ailleurs, que Freud a été le premier à mettre en doute la justesse du positionnement intérieur de l'analyste et à l'inviter à continuer son analyse à intervalles réguliers.

D'après A. Reich, pour que l'inconscient de l'analyste soit son instrument de travail, il faut que soient remplies certaines conditions. Ses pulsions inconscientes, ressorts profonds de son intérêt pour son travail, doivent être sublimées en talent psychologique. Par exemple, la curiosité de l'analyste, qui dérive habituellement d'un voyeurisme infantile, doit d'abord, pour être considérée comme une condition essentielle à l'analyse, être désexualisée, déconnectée des objets originels. Autrement, elle entravera l'évolution de l'analyse. En général, la distinction entre un bon fonctionnement de la sublimation et les divers types d'acting-out de l'analyste devra, selon elle, être très nette.

Il semble que A. Reich croit davantage à un moi de l'analyste, renforcé par l'analyse, débarrassé des parasitismes pulsionnels. Elle n'en rejette pas pour autant le contre-transfert, qu'elle le considère comme indispensable à une bonne analyse, tout en y mettant certaines conditions: «le contretransfert est une nécessité préalable à l'analyse. S'il n'existe pas, le talent et l'intérêt nécessaires font défaut. Mais, il doit rester dans l'ombre et à l'arrière-plan» (Reich, 1951).

Comment régler l'intensité de la participation de l'analyste dans sa relation avec l'analysant? A. Reich dispose de quelques outils qui semblent lui permettre de garder la juste distance. Elle parle d' «identification transitoire» de l'analyste au patient, afin d'éprouver lui-même les sentiments de l'autre et les forces instinctuelles sous-jacentes pour, ensuite, s'en détacher. Mais, dans ce processus d'empathie, l'identification de l'analyste se fait aussi à d'autres conditions: «Dans le cas de l'analyste, le processus d'identification et d'extraction comporte un investissement minimal de quantité d'énergie et doit avoir été précédé d'un processus de neutralisation très complet» (Reich, 1960). En cas contraire, l'analyste se trouvera trop impliqué et tout le processus aboutira à des manifestations contre-transférentielles, comme la réaction de l'analyste à l'impact des forces instinctuelles du patient par une réponse directe (non symbolique), ou bien à une incapacité d'abandonner l'identification au patient, en restant fixé dans le plaisir excessif que son identification lui procure. D'autres fois encore, l'analyste reverse ses propres 
sentiments sur ceux du patient, ce qui peut même déboucher sur un actingout transférentiel qui, justement, traduit ce revirement.

Il est clair qu'A. Reich n'a qu'une confiance très limitée dans le privilège du contretransfert dans la relation analytique et rejette son utilisation comme moyen d'explorer la situation du patient. Elle désapprouve l'idée que le contre-transfert est le retentissement, en l'analyste, des tendances et défenses qui prédominent chez le patient. De même, la tactique de l'école anglaise, qui voit le processus analytique comme une série d'identifications et de projections mutuelles entre l'analyste et le patient, lui semble inappropriée. Elle rejette en particulier le fait que les réponses émotionnelles de l'analyste au patient sont considérées comme identiques aux modèles originels de la période prégénitale (Deutsch, 1926), ce qui renvoie la remémoration et la reconstruction du passé à une place secondaire.

Nous voyons ici un autre style, un besoin de délimiter la relation entre analyste et analysant, une autre idiosyncrasie et, de toute évidence, d'autres patients. Pourtant, A. Reich considère de même avec scepticisme l'utilisation du contre-transfert lorsqu'il s'agit de patients « difficiles» (Little, 1951), tout particulièrement dans les cas où l'interprétation n'a aucun effet sur l'analysant. De même, elle doute des réponses émotionnelles de l'analyste au patient, même dans les cas de névroses sévères qui peuvent arriver jusqu'au développement d'une névrose de contre-transfert (Tower, 1956), afin de faire redémarrer le processus thérapeutique.

En général, A. Reich est en désaccord avec toutes les méthodes qui, donnant une place excessive à la participation affective de l'analyste, tendent au travail direct avec le ça et à l'influence directe sur les relations d'objet, abandonnant ainsi l'analyse de la pathologie du moi et de sa «rectification».

De la même façon, elle considérait qu'il fallait distinguer les manifestations de forces inconscientes des comportements et observations conscients adaptés à la réalité, et ce, tant chez l'analyste que chez l'analysant. Dans la situation psychanalytique, les deux participants ne sont pas seulement objets de transfert ou de contre-transfert, mais aussi objets de réalité. Elle disait qu'il ne faut pas surestimer le contre-transfert, parce qu'alors on entrave le déroulement de l'analyse. Il devrait en aller de même pour l'analyse didactique: il arrive également que l'intérêt du contrôleur se focalise sur la relation de l'étudiant avec lui, et non sur l'application des principes de la psychanalyse aux schémas complexes du matériel clinique. Il existe même une tendance à interpréter toute erreur du candidat comme une expression transférentielle, ce qui n'est pas toujours juste. 
En général, A. Reich a tenté, à sa manière, de montrer que ce qui se passe dans une psychanalyse ne concerne pas seulement la relation de l'analyste avec l'analysant, mais met aussi en jeu beaucoup d'autres choses. Si cette relation garde toujours son poids spécifique à travers tout le processus, elle ne doit toutefois pas le limiter.

La position adoptée par A. Reich révèle une analyste qui investit davantage dans le moi, le conscient et la réalité que dans le ça, l'affectivité et les pulsions. Venant d'une autre tradition analytique, influencée par Anna Freud et l'école américaine des relations d'objet, elle choisit une position qui, sans être désaffectée dans son lien au patient, exige néanmoins une limite. Les détails qu'elle nous fournit dans sa description des conséquences de l'implication affective et pulsionnelle de l'analyste dans sa relation avec son patient, démontre un énorme besoin de délimiter cet événement pulsionnel chez l'analyste tout autant que chez le patient. L'analyste, en fin de compte, devra faire une bonne analyse et y revenir régulièrement.

Qui désapprouverait cette proposition? Ce retour régulier, en tout cas, souligne une fois encore que les mystères et la dynamique de la vie psychique se dissimulent dans les intrications des forces pulsionnelles.

\section{Lucia Tower: la communication non verbale}

En 1932, la chirurgienne américaine Lucia Tower (1889-1981) quitte sa profession pour devenir la première étudiante et diplômée de l'Institut psychanalytique de Chicago, fondé par Franz Alexander.

Lucia Tower voit les phénomènes de contre-transfert comme une partie évidente de chaque processus de traitement et les considère comme inhérents à leur dynamique opérationnelle. La relation entre patient et analyste, dans la situation thérapeutique, se déploie à de nombreux niveaux, dont certains sont non verbaux. En ce cas, la relation se développe conformément au prototype de symbiose mère-enfant et, par le canal de la communication inconsciente non verbale, passent par des échanges libidinaux actifs qui les influencent l'un et l'autre. Ainsi, le contre-transfert est présenté comme une conséquence naturelle de la relation thérapeutique, exactement comme le transfert. Plus précisément, le contre-transfert «est la synthèse du moi inconscient de l'analyste, et, avec le transfert du patient, le produit du travail inconscient combiné du patient et de l'analyste» (Tower, 1955). De plus, «c'est un phénomène normal, produit par la compulsion de répétition». L'inter-action ou la trans-action du transfert avec le contre-transfert est présentée comme l'arrière-plan de la relation entre les deux protagonistes, 
comme le fond sur lequel sont exprimées les verbalisations intellectuelles et les interprétations. Cette communication à des niveaux inconscients peut s'avérer d'importance capitale pour l'issue du traitement.

Tower croit que la communauté psychanalytique manifeste une position négative envers le contre-transfert de l'analyste, lorsque, pour décider si un candidat convient à ce travail, elle attache une grande importance à ses ressources libidinales, qu'elle juge indispensables pour supporter le lourd poids des analyses intensives qui lui seront présentées. Or, le moindre indice d'investissement libidinal sur un patient est cependant banni comme dangereux, de sorte que reste ignoré un champ très propice à l'exploration et à la compréhension du processus analytique, à savoir le fonctionnement de l'analyste.

D’après son expérience, Lucia Tower juge naturel de rencontrer des phénomènes transférentiels chez tout analyste. Ces phénomènes possèdent des caractéristiques précises et frappantes:

- ils sont inhibés quant au but, ce qui signifie qu'ils ne poussent pas à l'action;

- ils sont extrêmement détachés des transferts érotiques du patient.

Elle ajoute que, dans de nombreux traitements analytiques intensifs, sinon tous, se développe chez l'analyste, en contrepartie inévitable de la névrose de transfert, une "structure de contre-transfert», qui ne s'identifie pas nécessairement à la névrose de transfert, bien qu'elle puisse l'être aussi. Cette formation est «le véhicule de la compréhension émotionnelle que l'analyste aura de la névrose de transfert» (Tower, 1955), et fait fonction de catalyseur pendant tout le processus. La compréhension de cette structure de contre-transfert chez l'analyste jouera un rôle important aussi bien dans la perlaboration finale de la névrose de transfert que dans la compréhension intellectuelle du transfert. Transfert et structure de contre-transfert sont deux forces qui ne cessent de s'influencer réciproquement au cours de l'analyse, et dont il ne faut pas négliger de tenir compte.

Pour ce qui est de l'analyste, bien qu'il s'efforce de se tenir sur la ligne droite de l'axe de son travail et de sa compréhension du cas, il semblerait, aux yeux de Tower, que les écarts, sous forme de petites modifications produites en lui en raison des pressions et motivations cachées de son patient, soient indispensables pour parvenir au grand changement qu'il espère luimême provoquer chez son patient.

Ainsi, Tower tient pour indispensable la participation de l'analyste à divers niveaux inconscients, étant donné qu'elle semble croire que c'est à 
travers eux que surgira l'élément qui causera des déplacements et des renversements tant chez l'analysant que chez l'analyste. De sa position se dégage une confiance dans cette tactique; elle n'hésite pas à demander une "compréhension émotionnelle», toujours dans l'esprit d'une mobilité de l'analysant dans l'analyste et vice versa, compréhension où l'analyste n'est pas pour autant dans la même position que l'analysant. Malgré tout, elle se concentre sur les ressources et les investissements libidinaux de l'analyste, considérant qu'il s'agit d'une dimension importante de la relation analytique: lieux dangereux pour la neutralité analytique ou, peut-être, données essentielles?

\section{Quelques déductions}

Nous avons examiné dans ses grandes lignes la position de quatre grandes dames de la psychanalyse, lesquelles, vers la même époque, ont élaboré, formulé et soutenu un discours important autour du contre-transfert. Qu'ont-elles en commun? Bien qu'elles proviennent d'horizons théoriques différents, elles se rejoignent audacieusement sur une même idée: le contretransfert est l'une des conditions nécessaires à la relation analytique et peut même devenir un outil précieux pour explorer l'inconscient de l'analysé. Ceci, naturellement, ne va pas sans risques. Chacune d'entre elles perçoit ces risques à sa manière et pose certaines conditions à son usage, lesquelles, néanmoins, ne devront en aucune façon éloigner l'analyste du vécu inconscient de son patient. Quels risques l'analyste prend-il donc en se laissant attirer dans le champ du contre-transfert? Pour Heimann, il risque d'être submergé par l'intensité des sentiments, tandis que Little craint la non-reconnaissance de ces sentiments. A. Reich reste dans une position d'extériorité par rapport à son patient et maintient le contre-transfert à l'arrière-plan. Quant à Tower, il lui suffira que le contre-transfert reste inhibé quant au but et détaché des transferts érotiques de l'analysant, de sorte qu'elle pourra même imaginer à l'avance ce qui pourrait advenir dans une séance future avec son patient. Qu'est-ce que cette nouvelle pratique analytique apporte? Il ne fait aucun doute qu'elle va à l'encontre de la fameuse position de neutralité dans laquelle l'analyste doit «se tenir». L'analysé "parle» à travers l'inconscient de l'analyste, il l'informe de ce qu'il n'est pas capable d'exprimer en mots. Bien entendu, l'identification projective, introduite dans la théorie analytique en 1946 par Melanie Klein, constituait en quelque sorte un outil conceptuel qui aurait pu expliquer de tels phénomènes de contre-transfert. Klein ellemême n'employait que rarement le terme de contre-transfert dans ses écrits théoriques. Cependant, certains pensent que toute sa technique est fondée 
sur ce concept (Mijolla et coll., 2002). Quoi qu'il en soit, pour Klein, c'est l'absence de réponse réelle de l'analyste qui donne à l'analysé la capacité de transférer. Par conséquent, il s'agit bien de l'émergence, au cours des années 50, d'une autre approche de la position de l'analyste, d'une autre clinique analytique dont nous pouvons discerner, depuis, l'influence sur diverses écoles analytiques à travers le monde, et cela jusqu'à nos jours.

Qu'est-ce qui a contribué à l'apparition de cette nouvelle tactique analytique? L'émergence et l'essor de la psychologie du moi, précisément à la même époque, semblent avoir considérablement contribué à orienter l'intérêt de nombreux psychanalystes vers la participation de l'analyste au champ analytique, en réaction à la méthode «classique» de rigueur aux États-Unis. L'analyste-miroir à l'attention flottante, toujours capable de prendre ses distances par rapport à la mémoire, la compréhension et le désir, cède dès lors sa place à l'analyste affecté d'un désir - moteur de la cure. Dans les années 50, les quatre analystes formulent, en quelque sorte, une «réponse » à l'orthodoxie ego-psychologique qui condamnait l'analyste à se figer dans une position de froideur technique, à consentir à d'innombrables séances silencieuses et, comme il était de règle, à éviter tout contact émotionnel avec l'analysé. Réagissant à leur manière aux tendances instaurées par l'école freudienne américaine, elles iront même jusqu'à identifier toute résistance aux résistances que l'analyste oppose à son contre-transfert. Et ceci est un point crucial. Car, un bon nombre d'années après Ferenczi et l'analyse mutuelle, elles attirent de nouveau l'attention des milieux analytiques sur le changement de dynamique que l'inconscient de l'analyste lui-même peut provoquer dans le processus analytique et, de surcroît, profondément convaincues qu'elles sont du bien-fondé de leur démarche, elles osent utiliser cette dynamique au profit de la cure.

Quel effet cette nouvelle proposition produit-elle? Elle fait passer au premier plan le désir de l'analyste qui, dès lors, s'affirme comme participation signifiante à la situation analytique. La question est de savoir comment il lui est possible de gérer cette participation. Et une autre de savoir s'il est capable de le faire. Le seul qui puisse répondre à ces questions est l'analyste lui-même, chaque analyste en tant que sujet distinct.

En effet, à notre avis, les voies de la relation duelle, les circonstances et les conditions d'une telle intersubjectivité entre l'analyste et l'analysé, pèsent différemment sur chacun. Les uns sentent qu'ils sont plus efficaces dans leur travail analytique quand ils adoptent une certaine position de «neutralité», en restant calmement à la place de l'Autre de l'analysant et 
laissant leur propre subjectivité hors du champ analytique. Or, ils traitent leur jouissance à l'aide de cette abstinence. Ils sont impliqués dans le champ du transfert de leur patient, mais à travers la seule «disparité» (Lacan, 19601), à une place différente que celle de leur analysant. Leur inconscient, purifié, devient transparent récepteur des messages venant de l'autre, messages qu'ils doivent «lire» et non pas ressentir.

D'autres, cependant, ne seront créatifs que s'ils se sentent libres de se mêler à l'inconscient de l'analysé, d'en accepter les messages au tempo relationnel et de les gérer à leur manière. Il s'agit d'ordinaire de ceux qui s'occupent de troubles cliniques plus graves - ce n'est pas une excuse, c'est une vérité. Se pourrait-il aussi qu'il s'agisse généralement de femmes? S'il en est ainsi, cela signifierait-il que l'inconscient féminin ait une plus grande facilité à passer par les voies de l' "amour", autrement dit celles du transfert et du contre-transfert, grâce à leur participation subjective, sans que cela ne coûte nécessairement ni à elle ni à l'analysé? La relation analytique, à travers le transfert, ressemble à celle de la première relation lointaine où « un tout savoir et un tout pouvoir de l'amour étaient les deux attributs accordés à ce premier représentant de l'Autre sur la scène de la réalité qu'a été la mère» ( ulagnier, 1988). S’amorce donc une relation de dépendance pour l'analysant, relation durant laquelle il va demander à l'autre d'occuper la position d'objet complémentaire. L'analyste-femme ne va pas, bien sûr, prendre la place de l'objet-complément pour la jouissance de l'autre (pour ne pas condamner son analysant à une telle place). Mais peutêtre pourrait-on dire qu'il lui est facile de se déplacer avec une familiarité provocante en réponse à l'autre qui demande un amour-complément, la femme, objet privilégié du désir qui se dévoile comme sujet du manque et qui peut offrir avec aisance, dans sa relation avec l'autre, sa non-identité du désir, dans laquelle, de toute façon, se construit la féminité (Aulagnier, 1967)? Bien que la femme soit le premier objet de dépendance, dispose-t-elle d'une souplesse qui, par l'«offre» de sa présence, inspire l'absence et le manque? Positions, métamorphoses et transsubstantiations à travers lesquelles il lui serait facile de passer, parce que seraient les voies du féminin...

Finalement, il revient à chaque analyste de choisir sa position en fonction de son tempérament, de son désir subjectif et de la confiance qu'il peut mettre dans une tactique clinique. Et c'est dans ces conditions que chaque analyste en vient à se mettre au service des tendances de son époque, qu'il tente de s'y adapter ou, au contraire, de les influencer.

Comme le disait Piera Aulagnier (1988), l'analyste durant son travail est confronté aux limites de ses possibles. L'éventail de ces possibles «dépend de 
notre capacité à nous mouvoir dans les positions identificatoires où nous projette notre partenaire, sans mettre en péril les repères garantissant à notre Je un principe de permanence sans lequel il ne pourrait donner place à un principe de changement». Le fonctionnement identificatoire de l'analyste doit être régi par ces deux principes s'il veut éviter «une position dépendante du droit d'occupation qu'un autre aurait seul le pouvoir de lui accorder ou de lui refuser».

\section{Daniella Angueli d.angueli@bbk.ac.uk}

\section{Références}

Angueli, D. (2010). Les résistances dans la cure psychanalytique. Paris: ANRT.

Aulagnier, P. (1988). Cent fois sur le métier on remet son écoute. Topique, 100 (3), 9-19.

Aulagnier, P. (1967). Remarque sur la féminité et ses avatars. Le désir et la perversion (p. 53-79). Paris: Seuil.

Balint, A. et Balint, M. (1939). On transparency and Counter-transference. Journal of PsychoAnalysis, 20.

Deutsch, H. (2000). Processus occultes pendant l'analyse. Les introuvables. Paris: Seuil.

Ferenczi, S. (1982). Euvres complètes, tome IV. Paris: Payot.

Heimann, P. (1949). Le contre-transfert. Paris: Navarin, 1987.

Lacan, J. (2001). Le transfert. Le Séminaire Livre VIII. Paris: Seuil.

Little, M. (1951,1956). Le contre-transfert. Paris: Navarin, 1987.

de Mijolla, A. et al. (2002). Dictionnaire international de la psychanalyse. Paris: Calmann-Lévy. Little, M. (1985). Lorsque Winnicott... Dans Transfert et états limites. Paris: Puf, 2002.

Reich, A. (1951). Le contre-transfert. Paris: Navarin, 1987.

Tower, L. (1955). Le contre-transfert. Paris: Navarin, 1987. 